\title{
Effects of Angle of Attack and Velocity on Trailing Edge Noise
}

\author{
Florence V. Hutcheson* \\ Thomas F. Brooks ${ }^{\dagger}$ \\ NASA Langley Research Center \\ Hampton, Virginia 23681-2199
}

\begin{abstract}
Trailing edge (TE) noise measurements for a NACA 63-215 airfoil model are presented, providing benchmark experimental data for a cambered airfoil. The effects of flow Mach number and angle of attack of the airfoil model with different TE bluntnesses are shown. Far-field noise spectra and directivity are obtained using a directional microphone array. Standard and diagonal removal beamforming techniques are evaluated employing tailored weighting functions for quantitatively accounting for the distributed line character of TE noise. Diagonal removal processing is used for the primary database as it successfully removes noise contaminates. Some TE noise predictions are reported to help interpret the data, with respect to flow speed, angle of attack, and TE bluntness on spectral shape and peak levels. Important findings include the validation of a TE noise directivity function for different airfoil angles of attack and the demonstration of the importance of the directivity function's convective amplification terms.
\end{abstract}

\footnotetext{
* Research Engineer, Aeroacoustics Branch.

$\dagger$ Senior Research Scientist Aeroacoustics Branch, Associate Fellow AIAA.
}

Copyright $(0) 2004$ by the American Institute of Aeronautics and Astronautics, Inc. No copyright is asserted in the United States under Title 17, U. S. Government has a royality-free license to exercise all rights under the copyright claimed herein for government purposes. All other rights are reserved by the copyright owner.

\section{INTRODUCTION}

Because of its importance to a wide range of applications (aircraft, cars, wind turbines, etc.) trailing edge (TE) noise is the subject of renewed interest. A number of theoretical and experimental studies have been conducted in the past five years.

From the theoretical side, Lummer et al. [1] developed a numerical model to study the influence of flow Mach number and non-linearities on the sound field generated by a single vortex passing the trailing edge of an airfoil placed at zero degrees angle of attack. Mach numbers between 0.2 and 0.5 were considered. It was found that the sound intensity scaled approximately as $\mathrm{M}^{4}$ downstream of the airfoil and that taking into account non-linearities could increase or reduce the sound pressure levels depending on the sign of the initial vortex disturbance. Singer et al. [2] simulated the acoustic scattering from vortices convecting past the trailing edge of a thin symmetrical airfoil, also at zero degrees angle of attack with Mach number varying between 0.2 and 0.4 . Velocity scaling of the computed acoustic signals indicated an approximate $\mathrm{M}^{5.2}$ dependency. Directivity patterns were compared to that of an acoustic source placed near the edge of a rigid half-plane. The two agreed generally well indicating a $\sin ^{2}(\square / 2)$ dependency of the acoustic intensity about the trailing edge. Other recent CFD studies include the use of Large Eddy Simulation (LES) for the prediction of TE noise. Manoha et al. [3] computed the noise radiated from a 3D lifting airfoil using a compressible threedimensional LES [4]. A NACA0012 airfoil placed at $5^{\circ}$ angle of attack and a flow Mach number of 0.205 was modeled. Wang and Moin [5] used LES to compute the TE noise from a two-dimensional flat strut with a beveled trailing edge and placed at $0^{\circ}$ angle of attack in a uniform flow of Mach number of 0.088 . 
Experimentally, Oerlermans, et al. [6], demonstrated that wind turbine noise reduction could be achieved through the use of optimized airfoil shapes and trailing edge serrations. They also developed a new microphone array processing method to enable noise source location on the rotating blades. Roger and Moreau [7] developed an analytical model to predict the noise radiated by a cambered airfoil at low Mach numbers. Their model is based on acoustical analogy and relates the far field acoustic pressure to the surface pressure near the trailing edge of the airfoil and to the statistical properties of the surface pressure field. Their model was validated by comparison to tests employing an instrumented cambered airfoil in low Mach number (below 0.1) and at different angles of attack. They used microphone probe sensors [8] to measure unsteady surface pressure. In a follow-up study [9], Moreau and Roger extended their experiment to include the effect of aerodynamic loading on the surface pressure spectra measured near the airfoil trailing edge.

With regard to providing benchmark experimental TE noise data, Hutcheson and Brooks [10] presented measurements from a NACA 63-215 airfoil model with different trailing edge configurations. Examined were spectra and directivity for clean and tripped boundary layer surface treatments. The results from only one test Mach number (0.17) and one airfoil angle of attack $\left(-1.2^{\circ}\right)$, nominal zero lift condition) were presented. To help interpret the results, corresponding semi-empirical predictions of Brooks, et al. [11] were presented.

The study of Ref. 10 emphasized measurement methodology for TE noise. The capabilities of two methods were evaluated via measurements of noise from the model airfoil, as well as from a cylindrical rod. The rod served as another example of the measurement of a uniformly distributed source across the test section sidewalls of an open jet. The first measurement method used a directional (or phased) array of microphones and the other was based on the cross spectral analysis of output signals from a pair of microphones placed on opposite sides of the airframe model (COP method). The microphone array was shown to accurately measure TE noise and to capture its two-dimensional characteristic over a large frequency range for any of the TE configurations tested, as long as noise contamination from extraneous sources was within bounds. The COP method was shown to also accurately measure TE noise but over a more limited frequency range that narrowed for increased TE thickness.

In the same time frame as Ref. 10, Oerlemans and Sijtsma [12] presented a study of the determination of absolute levels from phased array measurements, particularly of line sources (e.g. TE noise). The method proposed, after taking into account spatial source coherence, is to use an Array Calibration Function (equivalent to peak-output-level plots for line sources from Brooks and Humphreys [13]) to relate peak levels to total line (TE noise) source strength. This was in preference to applying the noise integration method of Ref. 13, because it was more straightforward for a line source, such as TE noise. The method proposed in Ref. 12 is similar in principle to that used in Mendoza, et al. [14] and in Ref. 10, with the exception that the underlying array processing of the method uses what is known as "diagonal removal" (DR) beamforming rather than standard (Std) array beamforming. The DR method is known to reduce signal-to-noise problems, but care has to be exercised in how the results are interpreted [13].

In the present paper, the study of Ref. 10 is extended to examine the important effects of flow Mach number and angle of attack on the TE noise radiated by the NACA 63-215 airfoil model with different TE bluntnesses. Far-field noise spectra and directivity are obtained using microphone array methods. Both DR and Std beamforming techniques are evaluated. The array outputs from both methods are scaled by a "perfoot" weighting function to put spectra on the same quantitative basis. The DR technique is used for the primary database because it is found to substantially overcome noise contamination problems due to flow recirculation over the face of the array. This contamination problem was not significantly present in Ref. 10, as only the nominal zero lift condition was reported. As in Ref. 10, TE noise predictions [11] are reported to help interpret the data and to also partially evaluate the application of the prediction method (which is based on symmetric NACA 0012 airfoil tests) to this cambered NACA 63-215 airfoil.

\section{TEST SET UP}

The trailing edge noise experiment was performed at NASA Langley Research Center in the Quiet Flow Facility (QFF), which is an open jet anechoic facility. The model used is a NACA 63-215 airfoil with a 16" chord and 36" span. The model (with a flap) is shown mounted in the test section of the QFF in Fig. 1. For this test, the flap was removed and the cutout for the flap was filled in and contoured to render a spanwise uniform trailing edge region. The model is supported above the 2 by 3 foot rectangular nozzle by two sideplates. Treatments were applied to the trailing edge of the airfoil to modify the thickness and to model blunt trailing edges, with either square or rounded corners [10]. As depicted in Fig. 2, seven trailing edge configurations were examined with the level of thickness " $h$ " varying from $h=0.13$ " to 0.005 ". 


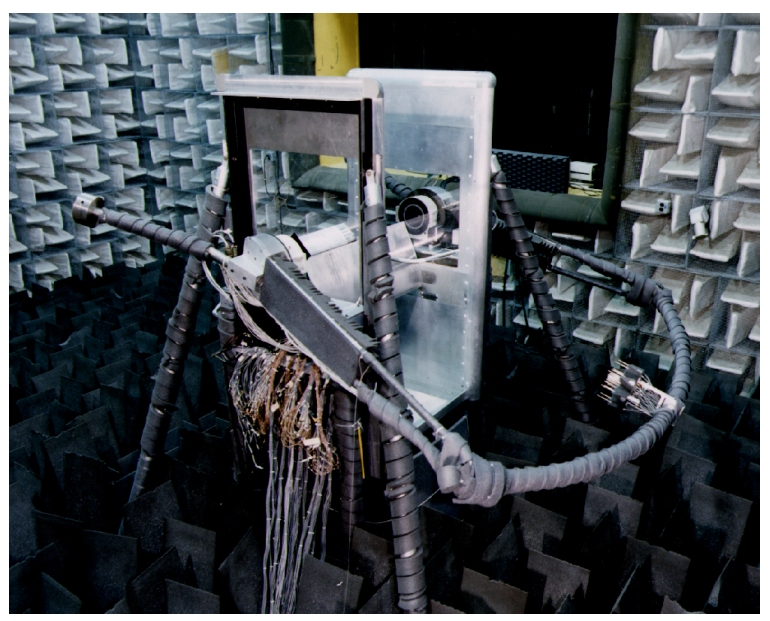

Figure 1. Test apparatus with phased microphone array mounted on pivotal boom in QFF.

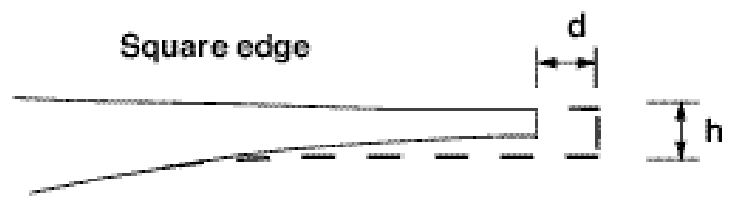

TE configuration \#1: $h=025^{\prime \prime}, \mathrm{d}=0$ (bascline) \#2: $\mathrm{h}=035 \mathrm{0}, \mathrm{d}=0$

*3: $\mathrm{h}=.075^{\prime \prime}, \mathrm{d}=043^{m}$

\#4: $\mathrm{h}=13^{\mathrm{m}}, \mathrm{d}=0$

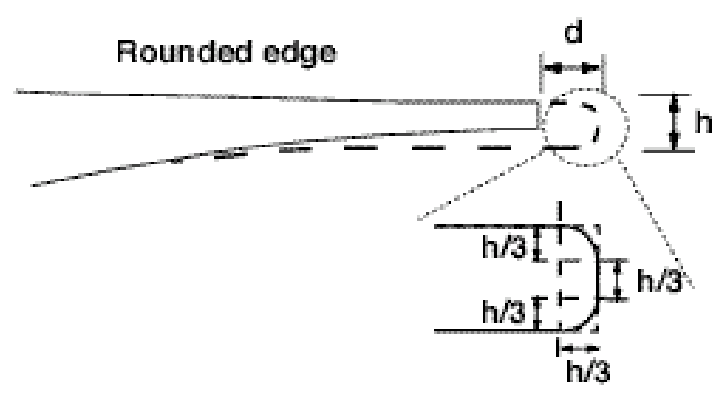

TE configuration $\# 5: h=075^{n}, \mathrm{~d}=, 043^{\prime \prime}$

\# $6 \mathrm{~s} \mathrm{~h}=13^{\prime \prime}, \mathrm{d}=043^{\prime \prime}$

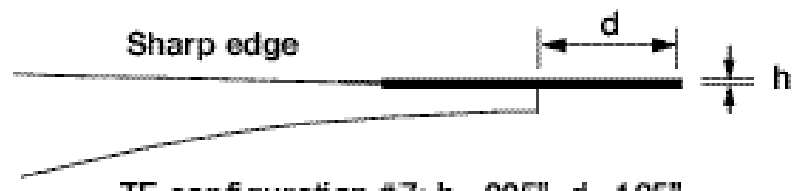

TE configuration \# $7: h=.005^{\prime \prime}, d=125^{\prime \prime}$

Figure 2. Airfoil trailing edge configurations
Grit, vortex generators or strips of serrated tape were used in certain configurations to trip the boundary layer (shown in Ref. 10). These leading edge treatments covered the first $5 \%$ chord region on the pressure and suction side of the model. In this paper, only the results obtained with \#90 grit leading edge treatment are presented.

The test conditions included mean flow Mach numbers ranging from 0.07 to 0.17 (corresponding to a wing Reynolds number ranging from about 0.6 to 1.6 million). The airfoil was placed at angles of attack ranging from $-6.2^{\circ}$ to $8.8^{\circ}$.

The measurements of the far field acoustics are made using the Small Aperture Directional Array (SADA) $[13,15,16]$. The SADA is composed of 33 B\&K 1/8" pressure microphones projecting from an acoustically treated frame. The aperture of this microphone array is small (7.78" in diameter), ensuring that all the microphones lie within approximately the same source directivity. As shown in Figs. 1 and 3, the SADA is mounted on a pivotal boom and can be placed around the model at different elevation angles $\square_{\text {SADA }}$. The SADA measurements are performed approximately five feet from the airfoil trailing edge, in the mid-span plane.

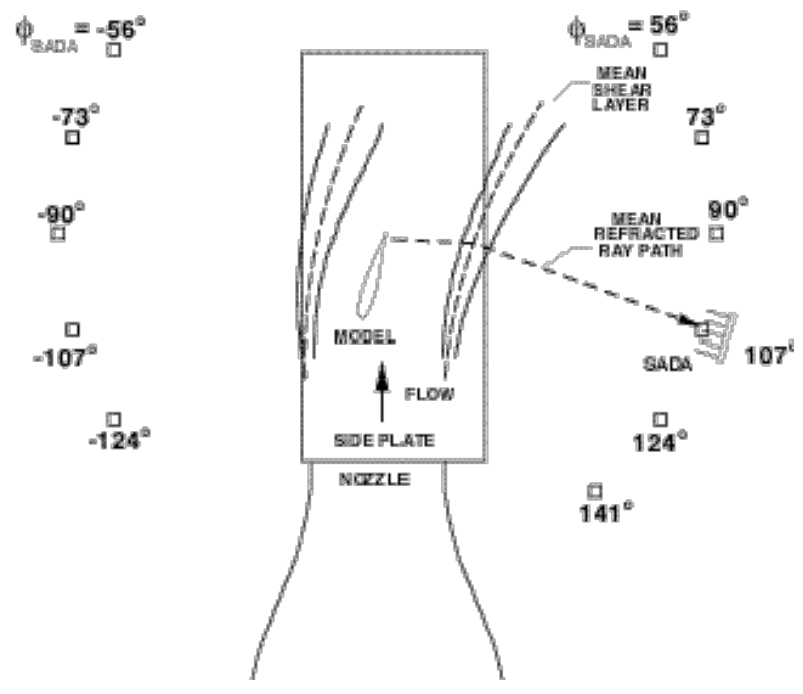

Figure 3. Sketch of the test set up with microphone array locations and elevation angles. 


\section{Data Acquisition and Processing}

Two transient data recorders (NEFF) controlled by a workstation were used for the data acquisition. The data from the 33 microphone channels were recorded simultaneously at a sampling rate of $142.857 \mathrm{kHz}$ and with a dynamic range of 14-bit. High pass and low pass filters, set respectively at $300 \mathrm{~Hz}$ and $50 \mathrm{kHz}$, were used to condition the outputs from each microphone channel. Piston-phone and injection calibrations of amplitude and phase were made for each microphone of the array $[15,16]$.

The cross-spectral matrix, $\mathbf{G}$, for each set of data acquired from the 33 microphones channels were constructed. Individual elements of the cross-spectral matrices were computed by partitioning each time signal into 1000 non-overlapping segments of $2^{13}$ samples. Each time history segment was then Fourier transformed using a Hamming window for signal conditioning. The resulting frequency resolution was $17.45 \mathrm{~Hz}$.

\section{ANALYSIS METHODOLOGIES}

\section{$\underline{\text { Microphone Array Methods }}$}

Both standard (Std) beamforming and Diagonal Removal (DR) methods are used to process the data [13]. With the latter method, beamforming is performed with the diagonal elements (the auto-spectra terms) of the cross-spectral matrix $\mathbf{G}$ set to zero. The DR method is used to improve the signal to noise ratio by removing microphone self noise contamination. In the present test, the noise contamination was caused by unwanted flow re-circulation that occurred over the face of the SADA. This flow re-circulation problem occurred for the test cases where the airfoil model was used and placed at angles of attack other than $-1.2^{\circ}$.

When standard (Std) frequency domain beamforming is used to electronically "steer" the SADA, the array output power spectrum for a chosen steering location is obtained from

$$
p(\mathbf{e})=\frac{\mathbf{e}^{T} \mathbf{W}\left(\mathbf{G} \square \mathbf{G}_{\text {background }}\right) \mathbf{W}^{T} \mathbf{e}}{\prod_{i=1}^{33} w_{i}}
$$

where $\mathbf{e}$ is the steering vector, the superscript $\mathrm{T}$ indicates complex transpose, $\mathbf{G}_{\text {background }}$ is the crossspectral matrix obtained from the data acquired when no model is present in the test section and $\mathbf{W}$ is a frequency-dependent weighting-function row matrix containing the $w_{i}$ weighting coefficients. $\mathbf{W}$ is defined such that the array beamwidth remains approximately invariant (and equal to 1 foot) with frequency between 10 and $40 \mathrm{kHz}[15,16]$.

When the diagonal removal (DR) method is applied, the output power spectrum is obtained from

$$
p(\mathbf{e})=\frac{\mathbf{e}^{T} \mathbf{W}\left(\mathbf{G} \square \mathbf{G}_{\text {background }}\right)_{\text {diag }=0} \mathbf{W}^{T} \mathbf{e}}{\prod_{i=1}^{33} w_{i}{ }_{i=1}^{23} w_{i}}
$$

In Eq. (2), the diagonal terms of the cross spectral matrices $\mathbf{G}$ and $\mathbf{G}_{\text {background }}$ are set to zero and the denominator is modified to accounts for the change in the number of terms in the cross spectral matrices.

Note that in Eq. (1) and Eq. (2), the steering vectors account for the refraction of sound transmission through the shear layer. The correction calculations [15] are based on Amiet's method [17], with modifications made to account for a curved three-dimensional mean shear layer surfaces.

The outputs from the two processing methods were compared for test cases where no flow re-circulation occurred and where the microphone signals appeared uncontaminated. The noise source distribution contours that were obtained when a calibrator source was placed in the flow at mid-span just above the airfoil TE are shown in Fig. 4. The airfoil was positioned at a $-1.2^{\circ}$ angle of attack. The calibrator source was the open end of a 1" diameter tube mounted to an out-of-flow acoustic driver. The input signal to the driver was white noise. Acoustic measurements were taken with the SADA located at an elevation angle of $107^{\circ}$ and aligned with the center of the trailing edge of the airfoil. The acoustic field contour maps presented were obtained by steering the array over a 5 ' by 5 ' plane centered at the calibrator source and containing the trailing and leading edges of the airfoil (the plane is off-vertical by $-1.2^{\circ}$ ). The flow is from bottom to top in the figure. At any point on the contour plot, the levels shown represent the output of the array when the array is steered to the point. The results obtained for the $6.3,12.5$ and $20 \mathrm{kHz}$ one-third octave bands using the Std beamforming and DR processing methods are shown. It is seen that with both methods, the array accurately locates the calibrator source and the same peak noise levels are obtained. With the Std beamforming method, the noise level falls as the array is electronically steered away from the calibrator source location. With the DR method, however, the noise level falls rapidly to $0 \mathrm{~dB}$, then become "ill-defined" with respect to a $\mathrm{dB}$ scale as the 
array is electronically steered away from the calibrator source location. These so-called "ill-defined" levels occur at point locations where the pressure-squared value calculated from Eq. (2) becomes negative. The occurrence of negative pressure-squared values will be explained subsequently.

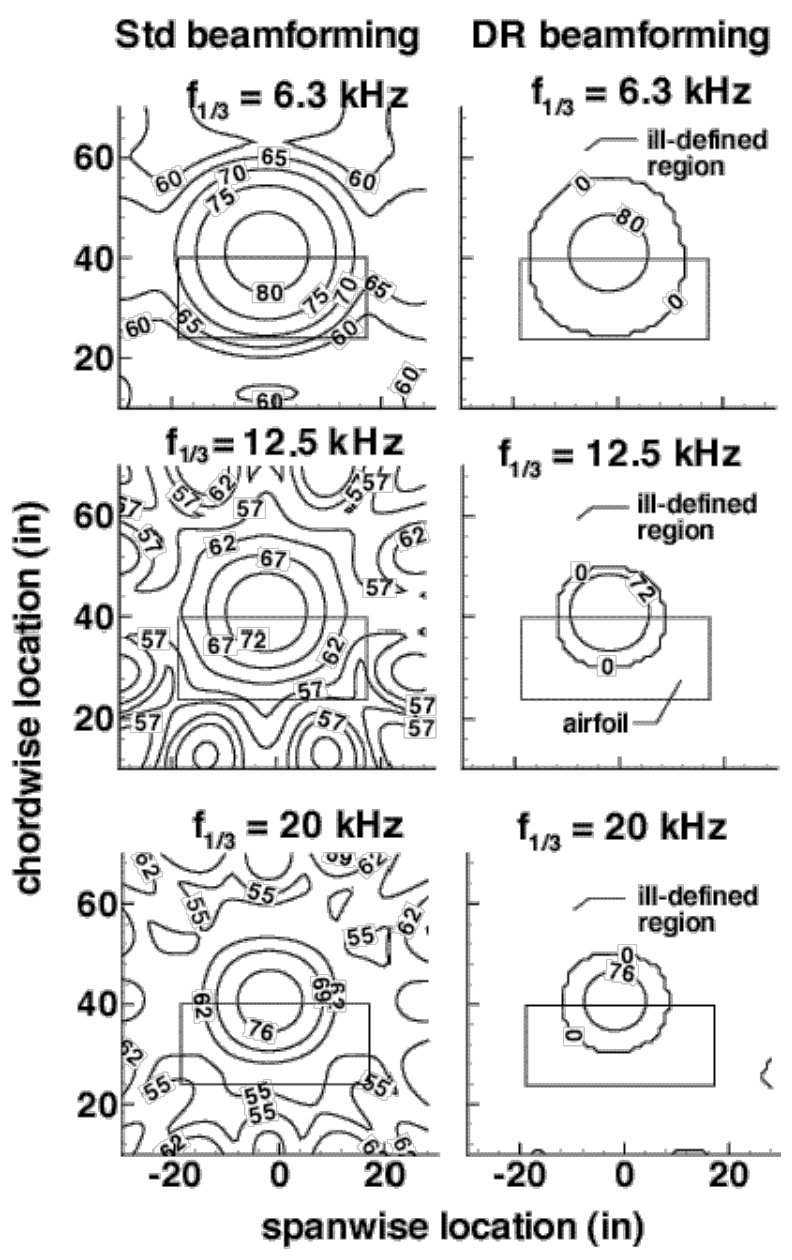

Figure 4. Noise source distribution contours over the pressure side of the airfoil at $-1.2^{\circ}$ angle of attack with a calibrator source located on the TE at mid-span. $M=0.17$, SADA elevation angle $\square=107^{\circ}$.

The noise source distribution contours that are obtained when the calibrator source is removed from the vicinity of the model's TE are presented in Fig. 5. The airfoil is still placed at $\mathrm{a}-1.2^{\circ}$ angle of attack and the TE configuration is TE\#7 (see Figure 2). Also shown in Fig. 5 are the maps obtained when the airfoil model was replaced by a 0.093 " diameter rod (the rod is positioned where the airfoil trailing edge was otherwise located). The results obtained for the $8 \mathrm{kHz}$, one-third octave band using the Std beamforming and DR processing methods are shown. It is seen that with both processing methods, the array successfully captures the two-dimensional characteristic of the noise radiated by the airfoil trailing edge and by the rod. However, the levels calculated using the DR method are lower by about $1 \mathrm{~dB}$ than the ones calculated with the standard beamforming method. As in the point source case, it is also seen that with the DR method the noise level falls rapidly to zero and becomes "ill-defined" as the array is electronically steered away from the TE noise source.

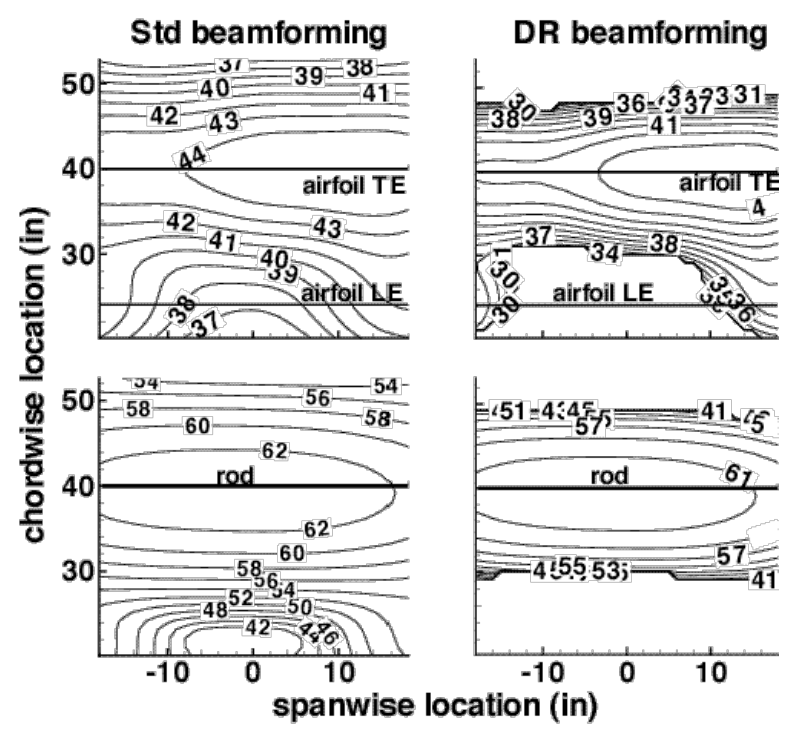

Figure 5. Noise source distribution contours for a $0.093^{\prime \prime}$ diameter rod and over the pressure side of the airfoil at $-1.2^{\circ}$ angle of attack with TE configuration \#7. $M=0.17$ and SADA elevation is $107^{\circ}$. One-third octave presentation. $f_{1 / 3}=8 \mathrm{kHz}$.

To explain the difference in the results obtained using the Std beamforming and the DR processing method, the theoretical SADA beam patterns that are obtained using the Std beamforming (Eq. (1)) and the DR methods (Eq. (2)) were studied. The two beam patterns are plotted in Fig. 6. The normalized pressuresquared levels are shown for a frequency of $8 \mathrm{kHz}$ over a 4 foot span. A point source is assumed to be located at the center of the scan line. It is seen in this figure that when the array is steered to the point source location, the pressure-squared level calculated using Std beamforming and the DR method match perfectly. However, when using the DR method, the pressuresquared level decreases to negative values as the array is steered away from the point source (leading to illdefined noise levels). With the Std beamforming method, the calculated pressure-squared values decrease, as expected, to values nearing zero. It is seen in Eq. (2) that the DR method's normalization only 
adjusts for response when focusing on a single point source. When focusing to locations along a distributed source, the levels for the DR method will be below those levels for the Std method due to integration over the response curves of Fig. 6.

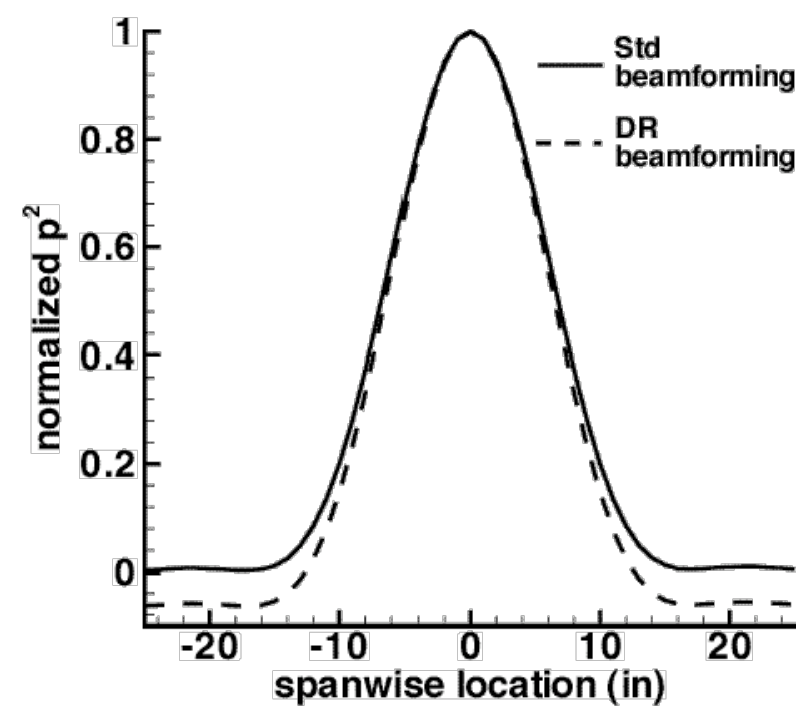

Figure 6. SADA theoretical output (beam pattern) response for a point source using Std beamforming and DR. The point source is located at the center of the scan line. $f=8 \mathrm{kHz}$.

\section{$\underline{\text { Per-Foot Presentation }}$}

In order to more clearly present the TE noise and rod noise measurements, the spectra were adjusted to represent noise generated on a per foot basis $[10,14]$ (i.e., the noise spectra represents that generated by a model of 1 foot span for an observer located five feet away). This was achieved by applying a frequency dependent weighting function to the noise level measured by the array when focused at the center of the line source. These functions for both processing methods are plotted in Fig. 7. The relatively flat portion of the functions near zero $\mathrm{dB}$ is representative of the fact that the SADA uses sub-array blending to achieve an effective one-foot beamwidth spatial resolution, independent of frequency, from 10 to 40 $\mathrm{kHz}[15]$.

The un-weighted and weighted (per-foot) spectra obtained using the Std and the DR processing methods for the test cases of Fig. 5, are shown in Fig. 8. It is observed that the levels from the weighted (per foot) TE noise and rod noise spectra are lower than that of the un-weighted spectra only below $10 \mathrm{kHz}$, where the main lobe beamwidth of the array is larger than 1 foot.

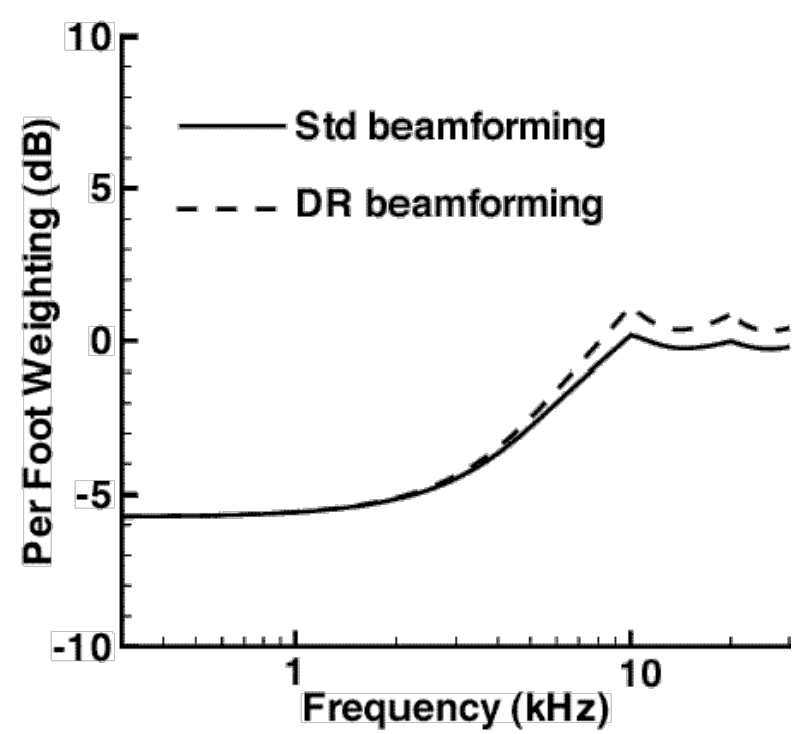

Figure 7. Per foot weighting function using standard and Diagonal Removal processing.

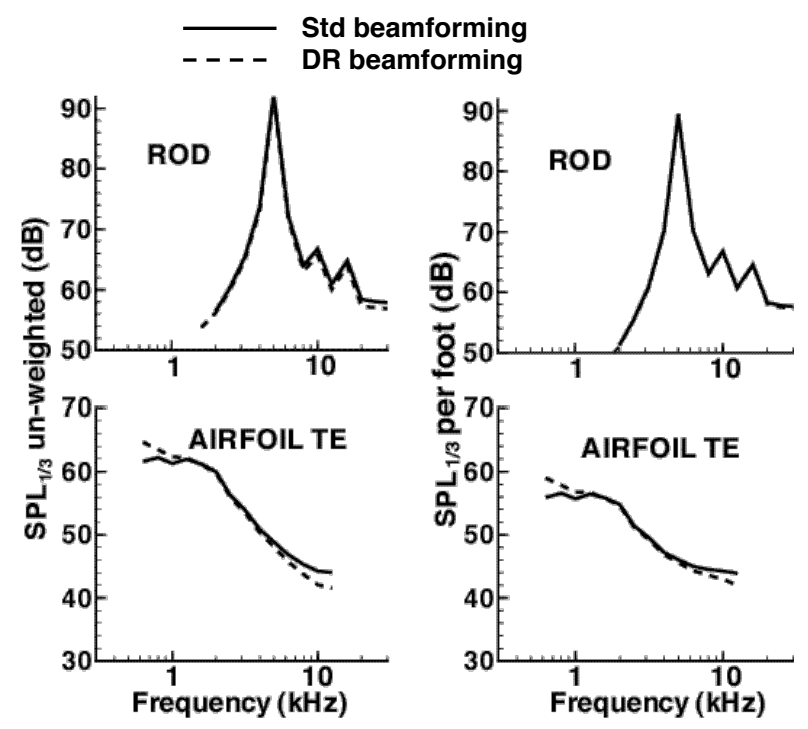

Figure 8. One-third octave band TE noise spectra for the airfoil at $-1.2^{\circ}$ angle of attack and for a $0.093^{\prime \prime}$ diameter rod. $\mathrm{M}=0.17$ and SADA elevation is $107^{\circ}$. Un-weighted and per-foot presentations.

It is also seen that the spectra for the two methods are better matched. For the rod case, they match closely. For the airfoil case, a difference in level of about $1.5 \mathrm{~dB}$ remains at $12.5 \mathrm{kHz}$. As it was explained in the previous paper [10], at (and above) $12.5 \mathrm{kHz}$, the noise levels measured at the TE are affected by the presence 
of strong noise about the leading edge region, due to its intrusion over the TE region by way of side lobes of the array. With the Std method, the LE noise can "add" to that measured over the TE. With the DR method, the LE noise can "subtract" from the measured levels over the TE. Hence, near $12.5 \mathrm{kHz}$, the correct TE noise level is between the ones calculated by the Std and the DR processing methods. Note that below about $1 \mathrm{kHz}$, for the airfoil TE noise case in Fig. 8, the level of the spectra obtained using the DR method is higher than that of the spectra obtained using Std beamforming. This is explained in the following section.

\section{Flow Noise Contamination}

For the test cases where the airfoil model was placed at angles of attack other than $-1.2^{\circ}$, flow recirculation occurred over the face of the SADA, when the SADA was located on the suction side of the airfoil. This is clearly seen by the presence of periodic patches of turbulence-related pressure fluctuations in the time history of the microphone signals (not shown here). A windscreen over the microphones would have eliminated the problem, which was not identified during testing. As will be shown, for these test cases, the recirculation affected the noise spectra over the entire frequency range of interest.

Noise contamination due to flow re-circulation also occurred (but to a much lesser extent) when the test models were removed from the test section in order to measure what is taken as the "background" noise. The background noise spectrum used in the computation of Fig. 8 is shown in Fig. 9. The TE noise spectrum obtained without subtracting the background noise is also shown.

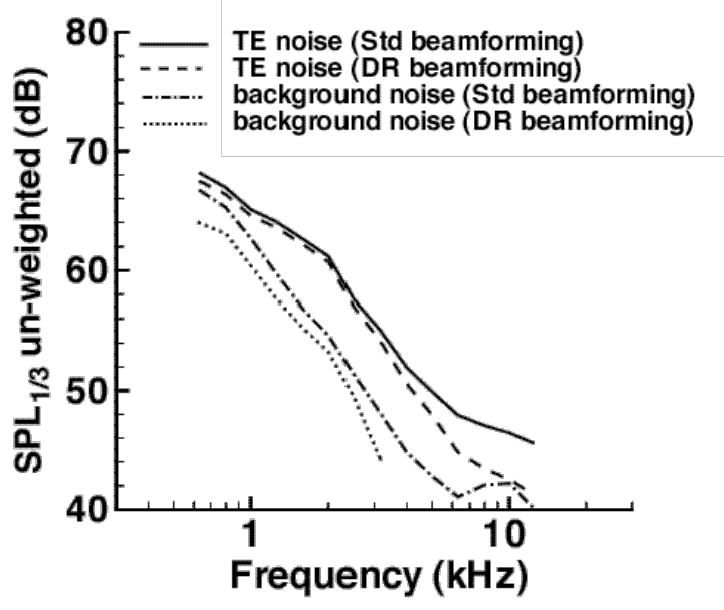

Figure 9. TE noise spectra (w/o background subtraction) for the airfoil at $-1.2^{\circ}$ angle of attack and background noise spectra. One-third octave band presentation, $\mathrm{M}=0.17$.
With Std beamforming, because of the noise contamination, the background noise level (below about $1 \mathrm{kHz}$ ) is nearly that of the TE noise. With the DR method, the microphone self noise is excluded, and the background spectrum remains lower in level than that of the TE noise (as it should be). Hence, when Std beamforming is applied, the background subtraction causes the level of the TE noise spectra to be lower than it should be in the frequency range where the background noise is contaminated. For the DR method, this problem does not occur because the noise contamination is removed from both the background and TE noise signals.

As mentioned, the noise contamination that occurs when taking array measurements on the suction side of the airfoil model, when placed at an angle of attack other than $-1.2^{\circ}$ could be extensive. Noise contour maps from the array for an angle of attack of $8.8^{\circ}$ are shown in Fig. 10. The results were obtained with the SADA at $+107^{\circ}$ and $-107^{\circ}$ locations. It is seen that on the suction side of the model (where noise contamination due to flow re-circulation occurs), elevated noise level are found using Std beamforming. The DR method removes the unwanted microphone self noise from the array processing, bringing the noise contour levels down. This is consistent with that found in Ref.12 for a somewhat similar setup. On the pressure

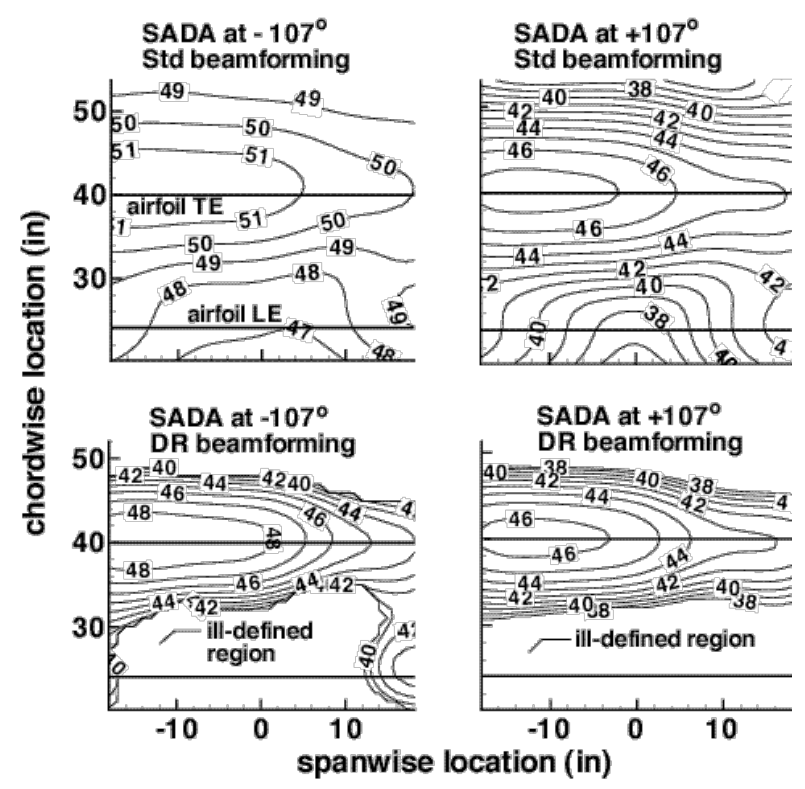

Figure 10. Noise source distribution contours over the pressure $\left(\mathrm{SADA}\right.$ at $107^{\circ}$ ) and suction (SADA at $-107^{\circ}$ ) sides of the airfoil using Std and DR beamforming. Airfoil is at $8.8^{\circ}$ angle of attack and $M=0.17$. One-third octave presentation with $\mathrm{f}_{1 / 3}=8 \mathrm{kHz}$. 
side of the model where the microphone signal are free of self noise, the noise contour levels obtained with both processing methods are of the same order (the ones obtained using the DR method being slightly lower than the ones obtained with Std beamforming, for reasons mentioned previously).

The corresponding per-foot spectra for TE noise are shown in Fig. 11. The effect of flow noise contamination is seen by the presence of increased levels when using Std processing for the suction side of the airfoil. Using the DR method, the noise contamination problem is overcome. The resulting spectrum is seen to agree very well with those using either method on the pressure side (where the microphones signals are uncontaminated). The DR method is used to obtain the results presented in the remainder of this paper.

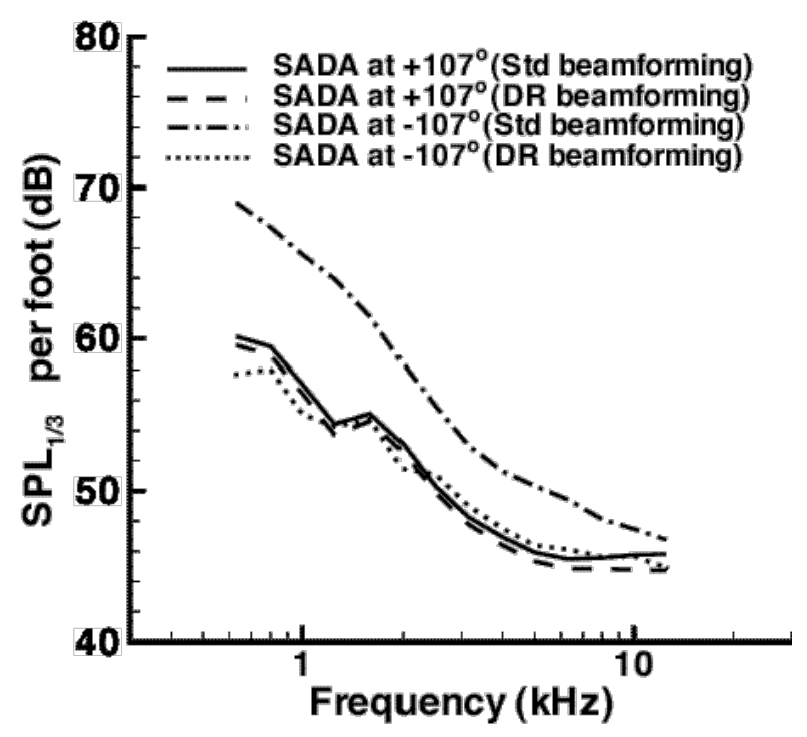

Figure 11. One-third octave band TE noise spectra for the airfoil at $8.8^{\circ}$ angle of attack and $M=0.17$. SADA elevation is $107^{\circ}$. Noise levels are on a per foot basis and adjusted to $90^{\circ}$ directivity.

\section{Comparative Prediction Method}

The airfoil TE noise prediction method compared to data is the one that was used in the previous paper [10]. It is a semi-empirical model [11] based on data acquired from aeroacoustic experiments by Brooks, et al $[18,19,20,21]$ using NACA 0012 airfoil sections. For the test cases presented in this paper, the boundary layer was fully tripped on both sides of the airfoil. Thus, the noise generated by the boundary-layer turbulence passing the trailing edge (TBL-TE noise) and the noise generated by vortex shedding from blunt trailing edges (BTE noise) dominate. This prediction method includes models of both sources.

The purpose of presenting comparative predictions is to help facilitate the understanding of the present data. The present results (including spectral shapes and parameter dependence) for the cambered NACA 63-215 airfoil are put in context with results (via the semiempirical predictions) for a reference airfoil. The reference NACA0012 airfoil has no camber and has a thinner thickness profile. To some extent, the comparisons can also serve to validate the prediction model; however, it is not straightforward on how to properly define corresponding inputs to the prediction code. The inputs should ideally account for all flow condition differences, of importance in the code, caused by the camber and thickness differences. However, for the limited purpose within this study, only approximate input adjustments are made.

The values calculated by the prediction code for the thickness $\square$ and displacement thickness $\square^{*}$ of the boundary layer at the TE of the airfoil are used for the predictions. Wind tunnel corrections are therefore required. The angle of attack of the airfoil is corrected to account for the deflection of the incident flow due to the finite size of the open jet. The corrected angles of attack $\square^{*}$ corresponding to the angle of attack in free air required to give the same lift as $\square$ would give in the tunnel are calculated from [11]

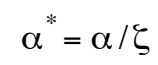

where

$$
\begin{aligned}
& \square=(1+2 \square)^{2}+\sqrt{12 \square} \\
& \square=\left(\square^{2} / 48\right)(\mathrm{c} / \mathrm{H})^{2}
\end{aligned}
$$

$\mathrm{H}$ is the open jet height ( 2 feet) and $\mathrm{c}$ is the chord of the airfoil (16 inches). More accurate corrections, which include camber effects, are contained in Ref. 21. However, it is not clear how an increased refinement with camber would be properly utilized here. For the present comparisons, a partially heuristic approach was taken to determine input angles. For the TBL-TE noise modeling, the above equations were used to obtain the angles $\square^{*}$, with $\square$ being simply defined as the geometric (waterline) angle in the test setup. These $\square^{*}$ values for the present test conditions are given in Table 1. For the BTE noise predictions, it was apparent by the noise results that the camber near the TE needed to be accounted for. This was done by simply adding $+2^{\circ}$ to the former $\square^{*}$ value, which matches the camber curvature between $x=(3 / 4 \mathrm{c})$ and the TE. Also, the value of the BTE parameter $\square$ in the code is taken as 
$15.5^{\circ}$, which is close to the value of $14^{\circ}$ found for the NACA 0012 airfoil. See Ref. 10 for an examination of sensitivity of inputs to predicted results.

Table 1. Geometric and corrected airfoil angles of attack used in this study for prediction comparisons.

\begin{tabular}{|c|c|c|}
\hline$\square$ & $\square^{*}$ (TBL-TE noise) & $\square^{*}$ (BTE noise) \\
\hline$-6.2^{\circ}$ & $-2.5^{\circ}$ & $-0.5^{\circ}$ \\
\hline$-1.2^{\circ}$ & $-0.5^{\circ}$ & $1.5^{\circ}$ \\
\hline $3.8^{\circ}$ & $1.6^{\circ}$ & $3.6^{\circ}$ \\
\hline $8.8^{\circ}$ & $3.6^{\circ}$ & $5.6^{\circ}$ \\
\hline
\end{tabular}

\section{Directivity}

In the results section, spectra obtained from SADA measurements at elevation angle $\square_{\mathrm{SADA}}$ are adjusted to represent the noise generated by a source of one-foot span, for an observer located five feet away and centered on the span. Further, the following radiation directivity function [11] is used to adjust spectral levels to correspond to an observer position at $90^{\circ}$ (normal to the chordline) in retarded coordinates.

$$
\mathrm{D}\left(\square_{\mathrm{r}}\right)=\frac{2 \sin ^{2}\left(\square_{\mathrm{r}} / 2\right)}{\left(1+\mathrm{M} \cos \square_{\mathrm{r}}\right)\left[1+\left(\mathrm{M} \square \mathrm{M}_{\mathrm{c}}\right) \cos \square_{\mathrm{r}}\right]^{2}}
$$

In Eq. (6), $M$ is the free stream Mach number, $M_{C}(\sim$ $0.6 \mathrm{M})$ is the convection Mach number assumed for boundary layer /wake turbulence convecting past the airfoil TE and $\square_{r}$ is the elevation angle of the SADA in the retarded coordinate system with respect to the airfoil chordline. $\square$, is defined in Fig. 12. In that figure, $\square$ is the airfoil angle of attack in tunnel coordinates.

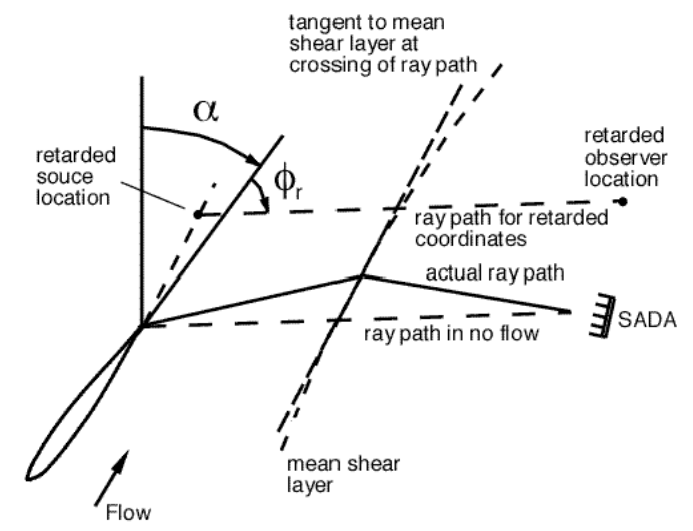

Figure 12. Ray path of TE noise propagating through the openjet shear layer and measured by SADA.
The directivity pattern of Eq. (6) is shown in Fig. 13 for a flow Mach number of 0.17 . The values of $\square_{r}$ corresponding to the different elevation angles $\square_{\mathrm{SADA}}$ of the SADA are indicated for an airfoil angle of attack of $-1.2^{\circ}$. It is seen that, for example, if the SADA is positioned at an elevation angle $\square_{\text {SADA }}$ of $107^{\circ}, \square_{r}$ is equal to $109^{\circ}$ and the $\mathrm{dB}$ correction to adjust the spectral levels to one of $90^{\circ}$ directivity is $-1.45 \mathrm{~dB}$.

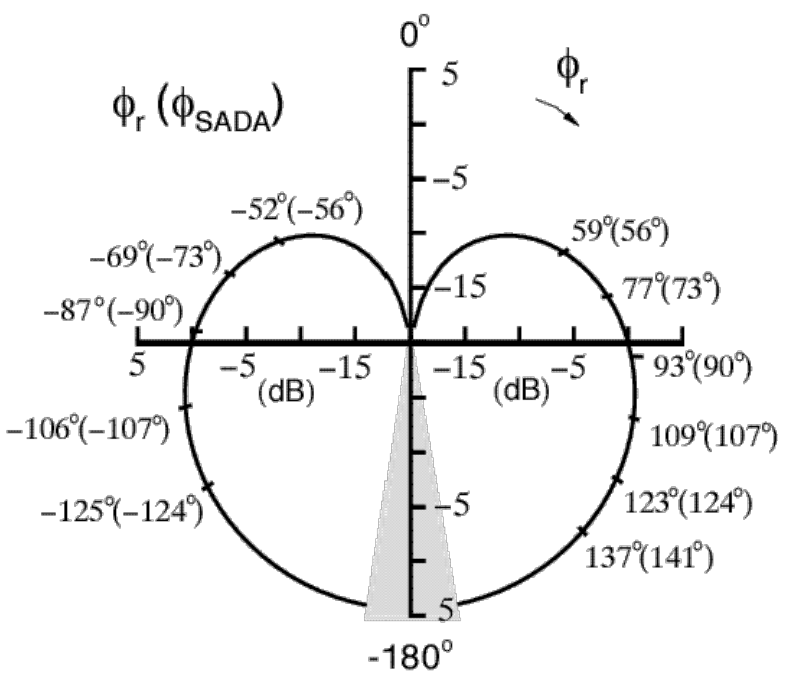

Figure 13. TE noise directivity function levels, with retarded

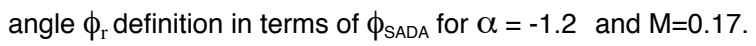

\section{RESULTS}

\section{$\underline{\text { Directivity }}$}

Trailing edge noise spectra for the airfoil at $\square=$ $1.2^{\circ}$ and $\mathrm{M}=0.17$, obtained from the SADA positioned at different elevation angles $\square_{S A D A}$ on both the suction and pressure sides of the airfoil, are given in Fig. 14. The airfoil TE configuration is \#7 ( $\mathrm{h}=0.005$ "). As for all spectra presented in this section, they are weighted on a per-foot basis using DR processing. In Fig. 15 (a), these same spectra are normalized to correspond to an observer positioned at $\square_{r}=90^{\circ}$ using the directivity function of Eq. 6. The values of $\square_{\text {SADA }}$ and the corresponding $\square_{r}$ are shown in Fig. 13 for this case. It is seen that with the exception of the more severe $\square_{s A D A}=141^{\circ}$ case, the spectra tend to collapse within several $\mathrm{dB}$. The same is generally true when we normalize the spectra for $\square=8.8^{\circ}$. This is strong validation of the TE noise directivity function Eq. (6). To examine this farther, the same spectra are normalized by Eq. (6) but with $\mathrm{M}$ set equal to zero (see Fig. 16). Here, the collapse is not nearly as good, 
indicating that the convective amplification terms involving $\mathrm{M}$ in the denominator are important and appear to correctly model the amplification effect.

Additional directivity normalizations, are shown in Fig. 17 for the same cases as Fig. 16 but where the Mach number examined is reduced to $M=0.11$. Here we see a similar success in normalization using Eq. (6), as was found in Fig. 15. It is noted that the effect of setting $\mathrm{M}$ to zero in the normalization was examined (although not shown here) in the same manner that was done for Fig. 15 in Fig. 16. A similar conclusion of the importance and correctness of the convective amplification modeling of Eq. (6) was found.

The results obtained for a blunt TE (configuration \#6, $\mathrm{h}=0.13$ ") are shown in Fig. 18 for the same two angles of attack. A similar success is found here as was found for the sharp TE in collapsing the spectra, even in the frequency range from 1 to $4 \mathrm{kHz}$ where BTE noise now dominates. It is also seen that the BTE noise peak frequency is independent of the directivity angle. Note that the spectra obtained with the SADA positioned at $-56^{\circ}$ elevation angle were omitted in most of these figures due focusing location questions related to extreme shear-layer/SADA angles.

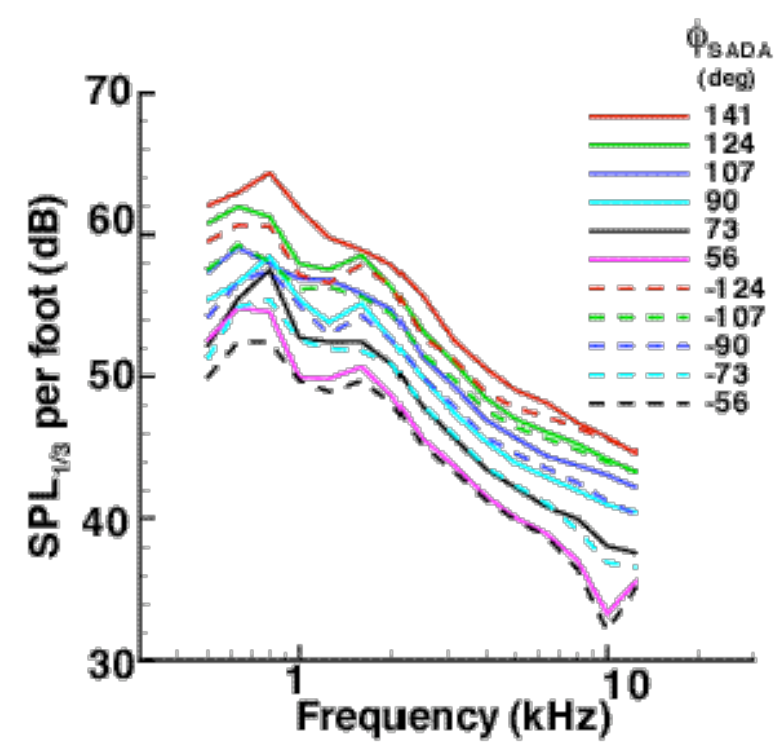

Figure 14. TE noise directivity. $M=0.17$; airfoil at $-1.2^{\circ}$ angle of attack; TE configuration \#7 ( $h=.005 ")$.
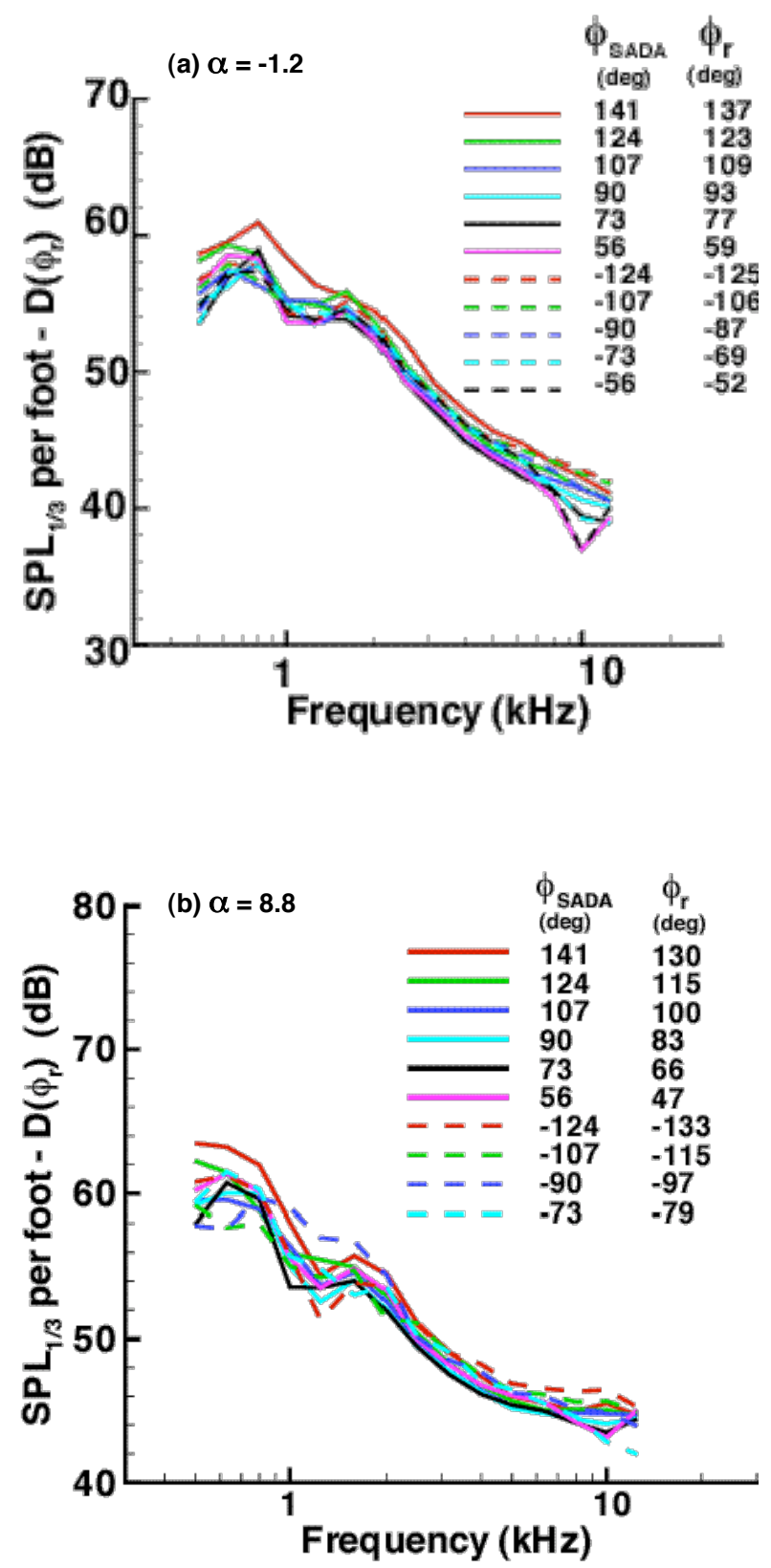

Figure 15. Scaled TE noise directivity. TE configuration \#7 $(h=0.005$ ); $M=0.17$. 

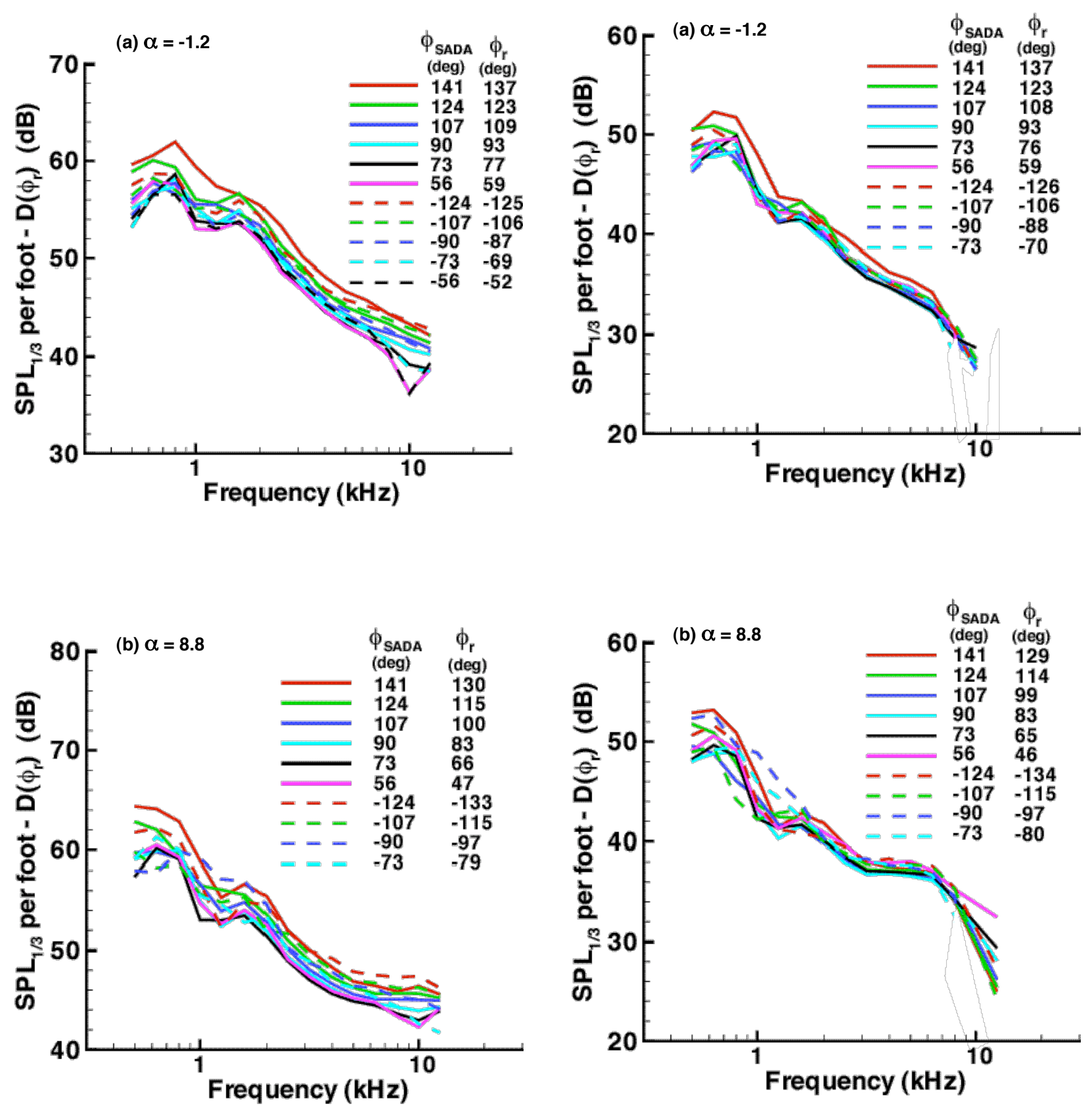

Figure 16. Same as Fig. 15, but scaled without convective amplification terms.

Figure 17. Scaled TE noise directivity. TE configuration \#7 $(h=0.005 ") ; M=0.11$. 

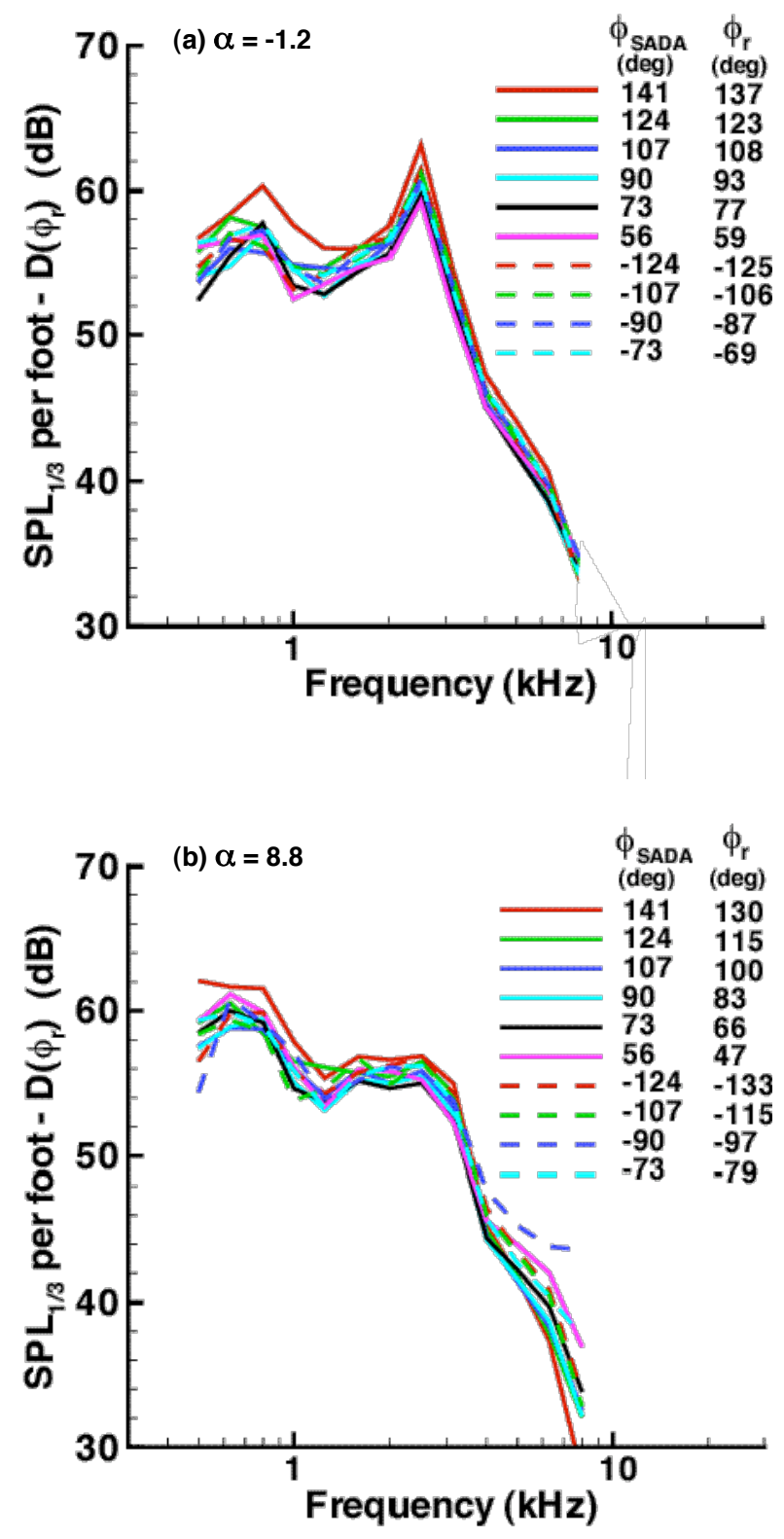

Figure 18. Scaled TE noise directivity. TE configuration \#6 $(h=0.13 ") ; M=0.17$.

\section{Effect of Flow Speed}

In order to more easily explain the behavior of the measured data, it is helpful to first introduce predicted results. Fig. 19 presents predicted TE noise spectra for the airfoil at $\square=-1.2^{\circ}$ and $8.8^{\circ}$ for different Mach numbers, corresponding to those experimentally tested. TBL-TE noise dominates the spectra over the whole frequency range shown. The spectra are presented on a per foot basis and normalized to $\square_{\mathrm{r}}=90^{\circ}$ (as with the experimental data). The spectra are additionally normalized in a simple Strouhal fashion where only Mach number is varied and a fifth power level dependence on velocity is assumed. It is seen that the spectra do not completely collapse (or coalesce). The predictions reflect the finding [11] that TBL-TE noise depends, in addition to Mach number, on boundary layer displacement thicknesses and Reynolds number effects on both the pressure and suction surfaces.
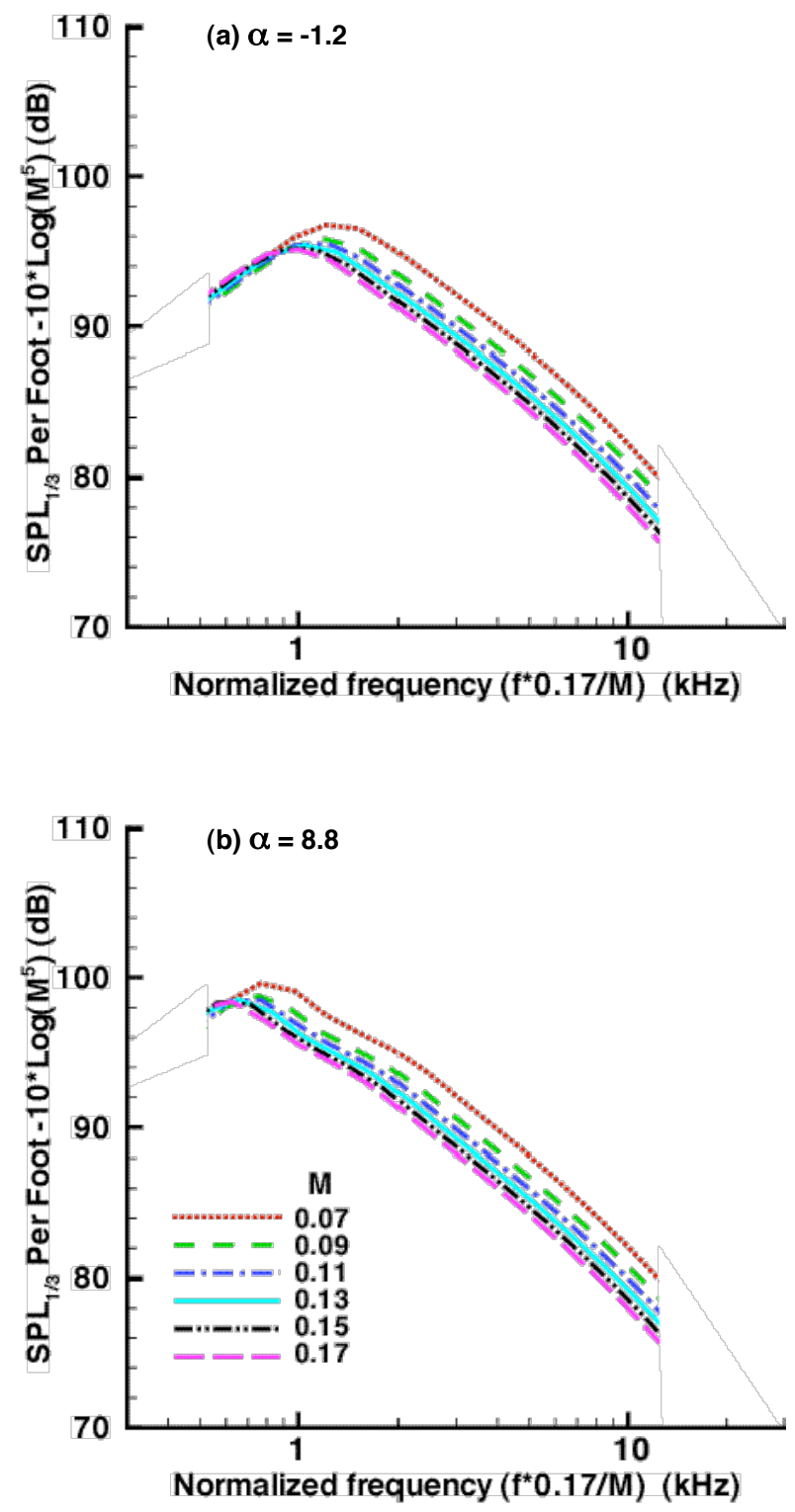

Figure 19. Effect of flow speed. TE configuration \#7 ( $h=0.005 ")$. Prediction. 

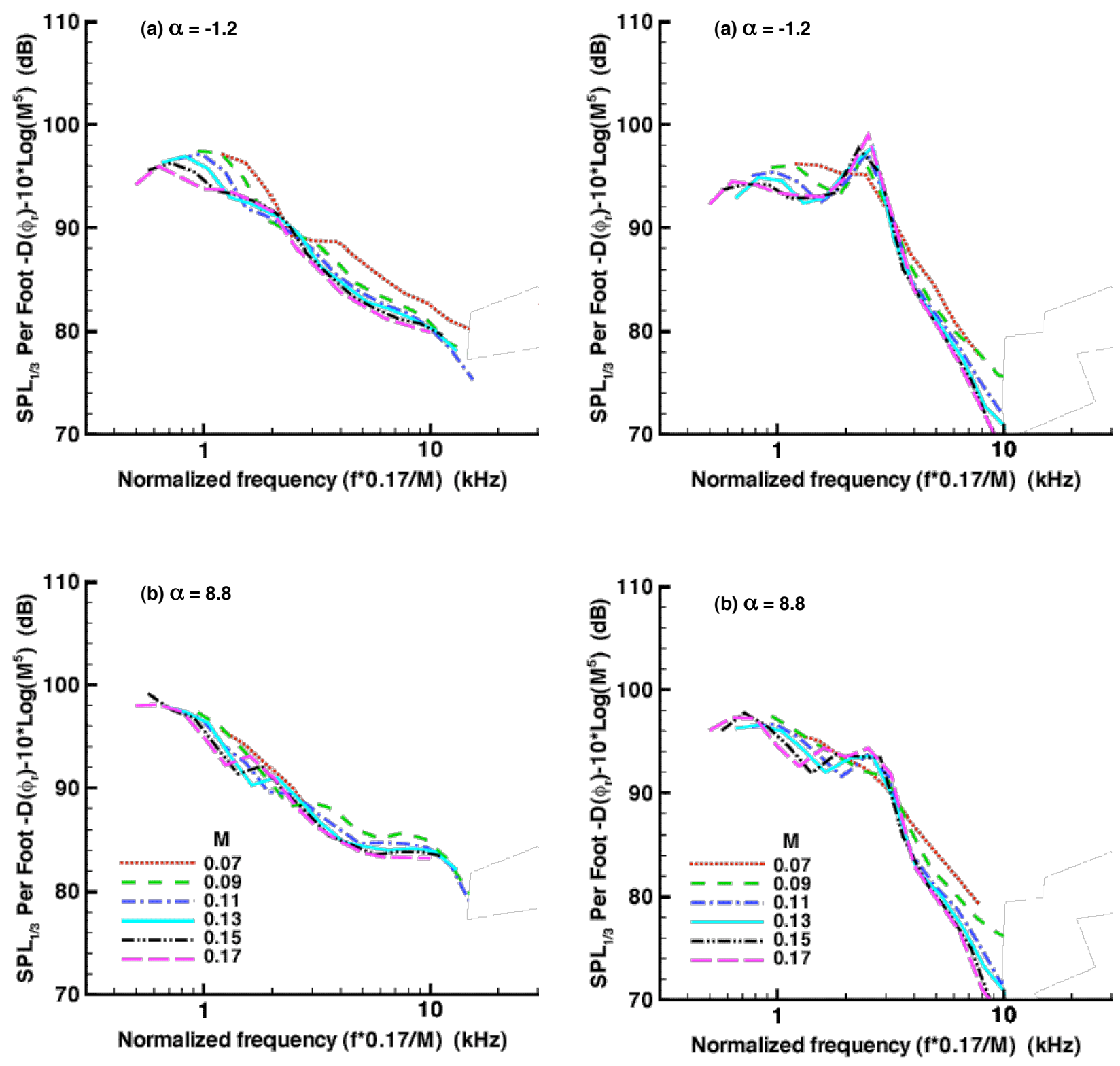

Figure 20. Effect of flow speed. TE configuration \#7 ( $h=0.005 ”)$.

Figure 21. Effect of flow speed. TE configuration \#6 ( $h=0.13 ")$

Fig. 20 shows the corresponding measured spectra for the same airfoil conditions as that predicted in Fig. 19. As is the case for the remaining spectra in this report, the spectra were obtained at $\square_{\mathrm{SADA}}=107^{\circ}$, and normalized in level at $\square_{r}=90^{\circ}$. The normalized spectra are seen to match that predicted, in basic ways. The lower speeds have the same spectral shift to higher levels and frequencies. The peak levels, peak frequencies, and fall-off in level with frequency generally match. There are differences, such as the spectral hump near $10 \mathrm{kHz}$. This may be BTE noise for

this $\mathrm{h}=0.005$ " bluntness, but the predicted frequency is higher than that observed here.

Fig. 21 shows measured spectra for the same conditions except the airfoil has a blunted TE configuration \#6 ( $\mathrm{h}=0.13$ "). The same general spectra behavior with speed is found here as was noted for Fig.20 for the sharper TE, except here BTE noise is present and its scaled levels are reduced at the lowest speeds. Corresponding predictions for Fig. 21 are not shown, but limited blunted TE cases are considered subsequently. 


\section{Effect of Airfoil Angle of Attack}

Predicted spectra for the different airfoil angles of attack are given in Fig. 22. TE configuration \#7 $(\mathrm{h}=0.005$ ") and a flow Mach number of 0.17 are modeled. With increasing airfoil angle, it is seen that although the spectral peak shifts to lower frequencies and its level increases, the higher frequency fall-off portion of the spectra is seemingly invariant. This appearance is due to [11] increased low frequency contributions from the suction side of the airflow because of its thicker boundary layer thickness (with larger turbulence scales) and an increased higher frequency contribution from the pressure side due to its thinner boundary layer thickness (with small turbulence scales). The increased noise contribution to TE noise from the pressure side serves to offset the loss of high frequency content from the suction side.

Measured spectra, matching those of the predicted cases of Fig. 22, are shown in Fig. 23 (a). There are detail differences in spectral shape compared to the predictions, particularly for $\square=-6.2^{\circ}$ near $1 \mathrm{kHz}$ and for $\square=8.8^{\circ}$ at higher frequencies. Still, the levels and the dependence on angle of attack basically agree. The results obtained with increased bluntness at the TE are presented in Figs. 23 (b), (c), and (d) for $h=.025$ ", .075 ", and .13", respectively. It is seen in these figures

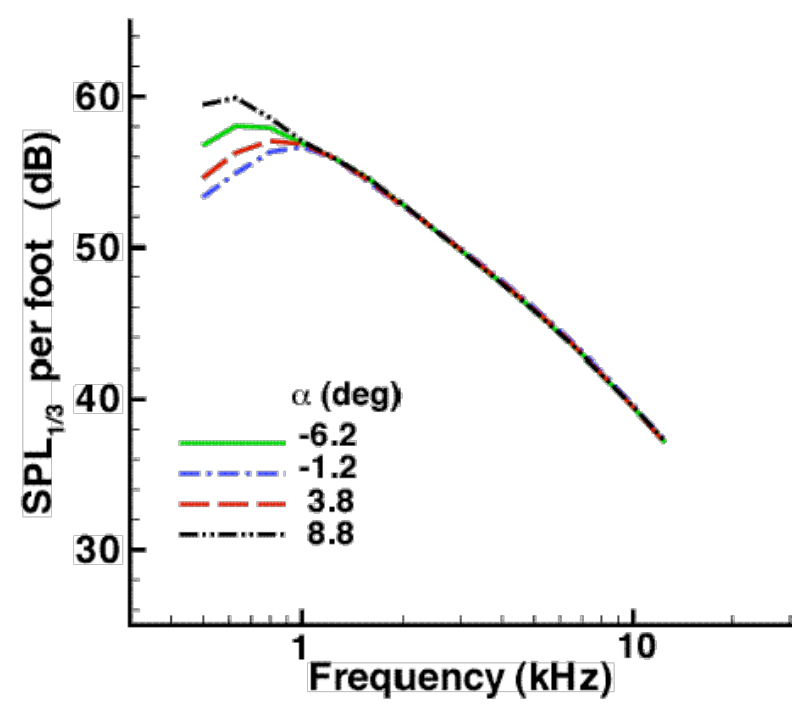

Figure 22. Effect of angle of attack. TE configuration \#7 $(h=.005 ") ; M=0.17$. Prediction. that the lower frequency behavior with changes in angle-of-attack appears to be little affected by the bluntness differences. However, the BTE noise spectral peaks, which become more prominent with increased bluntness, are indeed affected by angle of attack. The larger the angle, the more reduced the spectral peaks. This is discussed subsequently.

\section{Effect of TE Bluntness}

Predicted spectra, including the effect of bluntness, are presented in Fig. 24 for the two angles, $\square=-1.2^{\circ}$ and $8.8^{\circ}$. The prediction for BTE noise is seen to be simply superimposed on the prediction for TBL-TE noise for the total TE noise prediction. As indicated in the methodology section, the input definition for the BTE is not established for a cambered airfoil. The choices of $\square^{*}$ in Table 1 and that of $\square$ are regarded as heuristic. Still, the prediction comparisons are seen to lend greatly to the interpretation of the measurement results.

BTE noise scales with Mach number $M$ and Strouhal number (frequency $\cdot \mathrm{h} / \mathrm{U}$ ). It also depends [11] on TE thickness $h$ compared to the boundary layer thicknesses and TE geometric features (such as roundness) that determine flow angulation in the separated region aft of the TE. It is seen in Fig. 24 (a), for small angle of attack $\square$, that the larger thicknesses "h" produces a higher level at a lower frequency. For decreasing thickness $\mathrm{h}$, the frequency increases. Also, BTE levels diminish and spectra broaden since $h$ compared to boundary layer thicknesses decrease. For the larger $\square=8.8^{\circ}$ angle (of Fig. 24 (b)), the boundary layer thicknesses increase, which leads to diminished levels of the BTE noise.

In Fig. 25, measured spectra are shown that correspond to the predicted cases of Fig. 24. The general features are well matched between the figures. However, the levels of the BTE noise spectra humps differ somewhat. The spectral drop-off at frequencies higher than the spectra humps fall off more rapidly than predicted. This was noted in Ref. 10 for similar prediction comparisons. To show the effect of rounding the TE of the airfoil, the measured spectra obtained for TE configurations \#5 and 6 are also given in Fig. 25. It is observed that a rounded TE leads to a broader BTE noise spectral peak without significantly affecting its amplitude nor its peak frequency. This is also observed in Fig. 26, where the spectra obtained with a flow Mach number of 0.11 are displayed. 

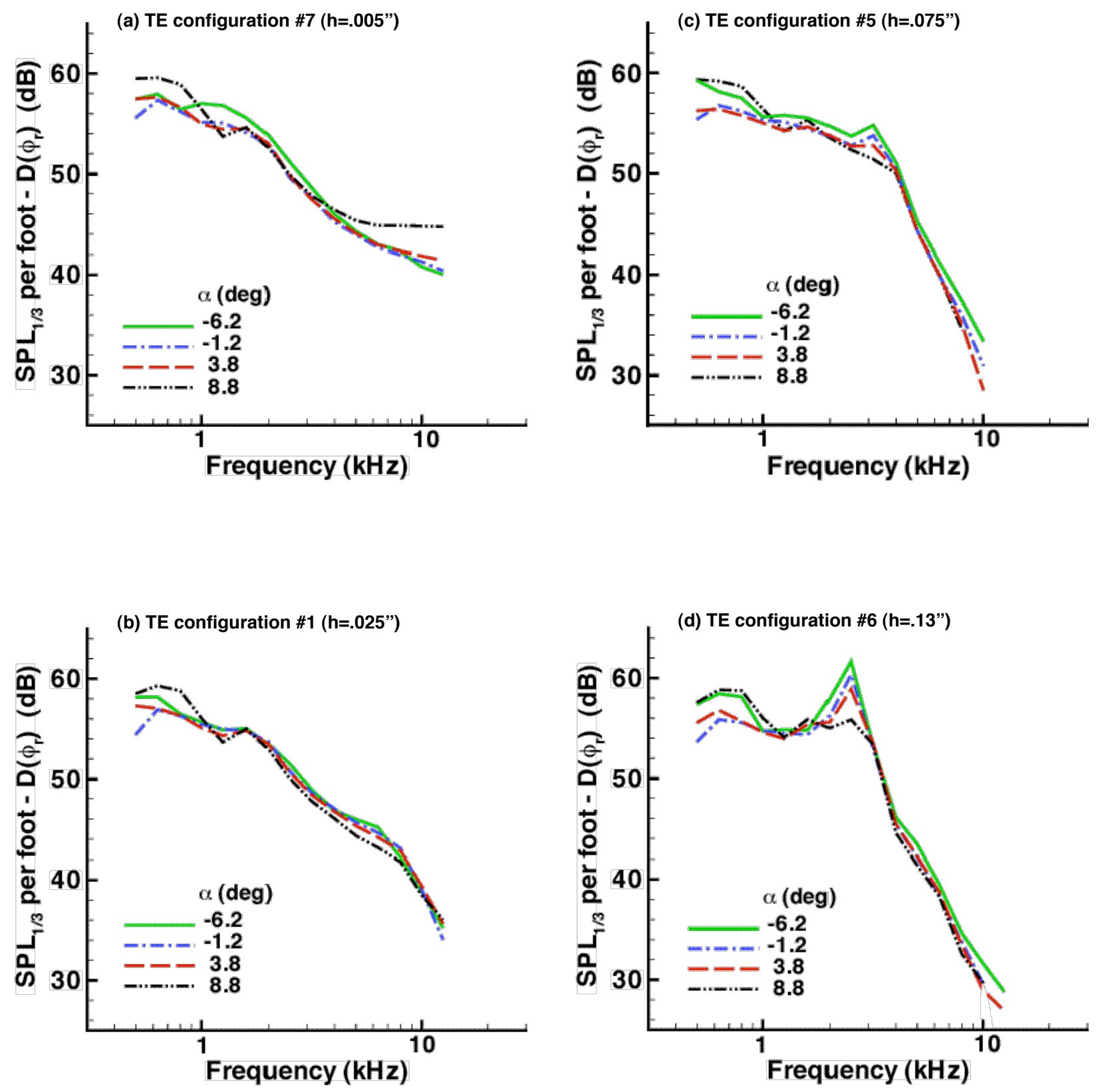

Figure 23. Effect of angle of attack. $M=0.17$. 

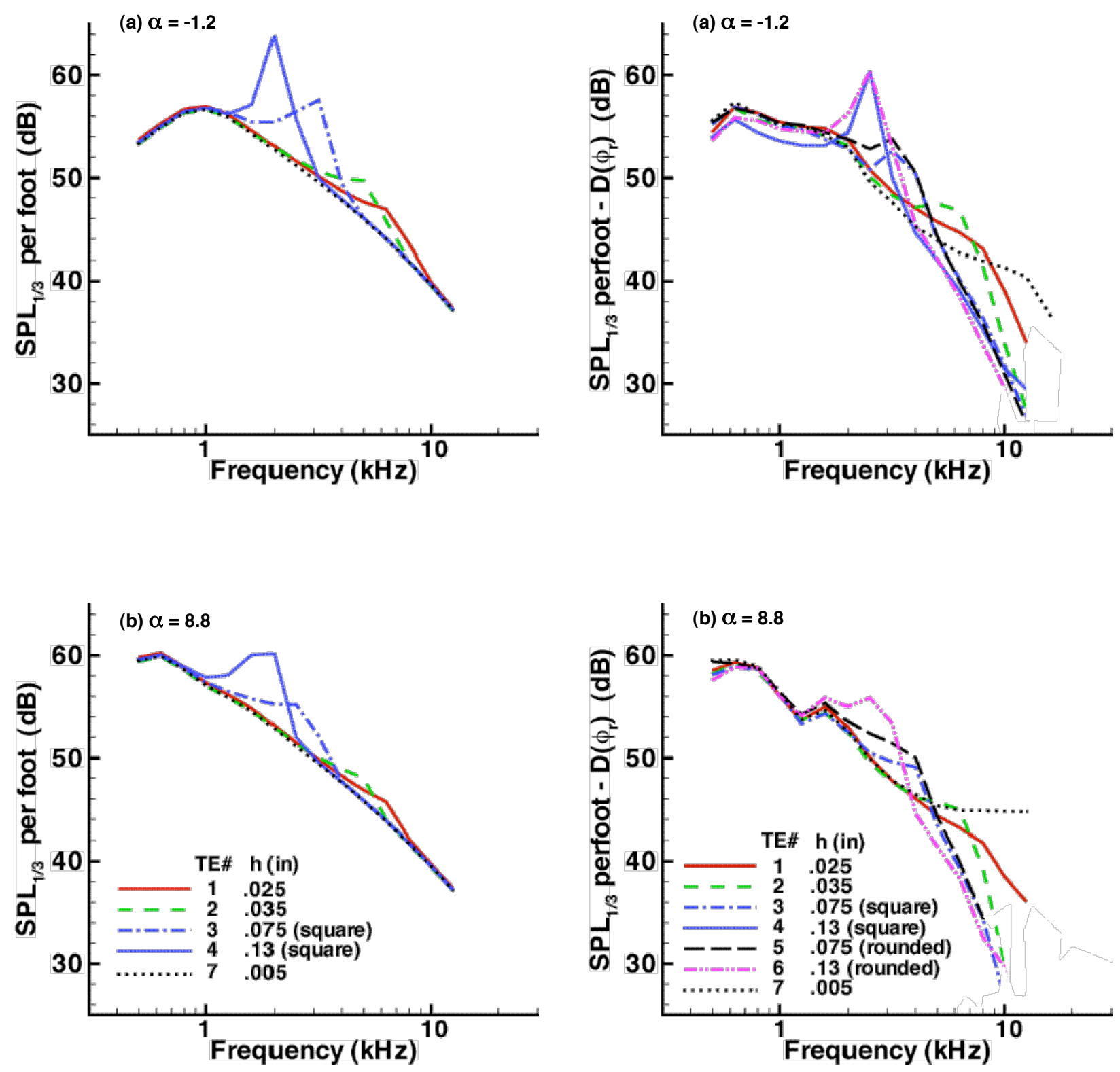

Figure 24. Effect of TE bluntness. $M=0.17$. Prediction.

Figure 25. Effect of TE bluntness. $M=0.17$. 

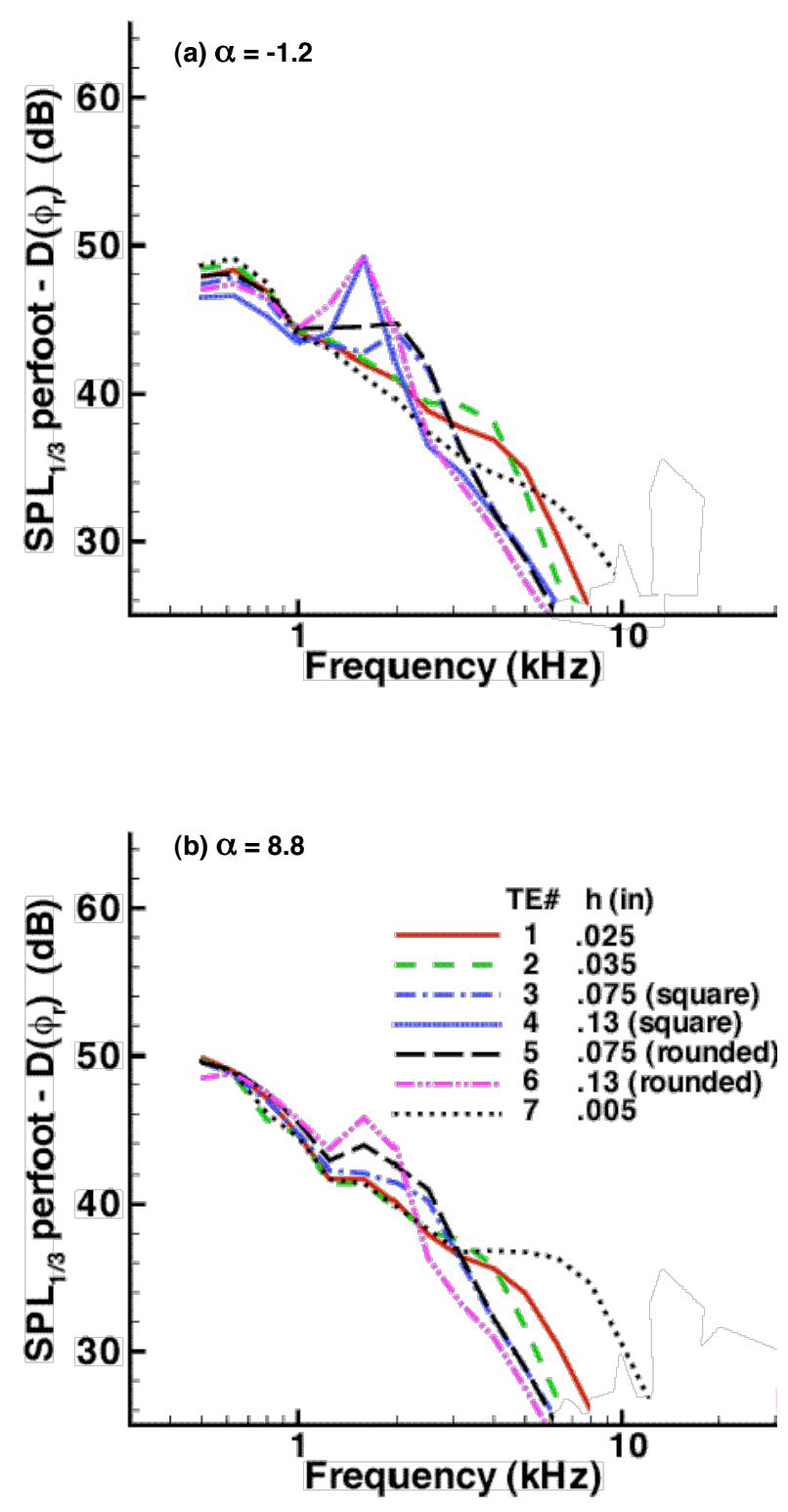

Figure 26. Effect of TE bluntness. $M=0.11$.

\section{CONCLUSIONS}

Trailing edge (TE) noise measurements for a NACA 63-215 airfoil model are presented. Far-field noise spectra and directivity are obtained using a phased microphone array.
The use of Standard (Std) and Diagonal Removal (DR) beamforming techniques are evaluated for this distributed noise problem. It is shown that the output from the DR method matches that of the Std processing only when focusing on a single point source. When focusing along a distributed source, the levels of the spectra obtain with the DR method are below those obtained with the Std method. The DR method also produces non-physical negative pressure-squared values when focusing away from the source. Using tailored weighting functions, the outputs from the two methods can be corrected such that each accounts for the distributed line character of TE noise. The corrected spectra obtained from each method match as long as other sources do not contaminate the measurements. Since the DR method is found to successfully discriminate against flow noise contamination, it is used to establish the primary database of this study.

The primary purpose of the study is to examine the effects of flow Mach number and angle of attack on the far-field spectra and directivity of the TE noise radiated by the cambered airfoil model with different TE bluntnesses. The interpretation of the measured results is greatly aided by comparative predictions. Although the semi-empirical predictions are based on a symmetrical airfoil and code inputs for the present cambered airfoil are not established, the predictions in most respects followed the measurements closely in level and spectra character. Key findings of this study include:

(1) A predicted TE noise directivity function is validated over broad directivity angles and for different airfoil angles of attack. The importance of the convective amplification terms is demonstrated.

(2) For increased airfoil angle of attack, the spectral peak of Turbulent Boundary Layer (TBL) related TE noise increases in level and shifts to lower frequencies, while the Blunt (B) TE noise spectral peak level decreases;

(3) For increased air speed, BTE and TBL-TE noise spectra increase in level and shift to higher frequencies;

(4) For increased blunt TE thickness, BTE noise spectral peak increases in level and shifts to lower frequencies; rounding the TE broadens the spectral peak.

\section{ACKNOWLEDGMENTS}

The authors wish to thank their colleague William M. Humphreys for his valuable assistance in the data processing. 


\section{REFERENCES}

1. Lummer, M., Delfs, J. and Lauke, T., "Simulation of Sound Generation by Vortices Passing the Trailing Edge of Airfoils", AIAA 2002-2578.

2. Singer, B., Brentner, K., Lockard, D. and Lilley, G., "Simulation of Acoustic Scattering from a Trailing Edge", AIAA 99-0231.

3. Manoha, E., Delahay, C., Sagaut, P., Mary, I., Khelil, S. and Guillen, P., "Numerical Prediction of the Unsteady Flow and Radiated Noise from a 3D Lifting Airfoil”, AIAA 2001-2133.

4. Manoha, E., Troff, B. and Sagaut, P., “Trailing Edge Noise Prediction using Large Eddy Simulation and Acoustic Analogy", AIAA 981066.

5. Wang, M. and Moin, P., "Computation of TrailingEdge Flow and Noise Using Large Eddy Simulation", AIAA Journal, Vol.38, No. 12, December 2000.

6. Oerlemans, S., Schepers, J. G., Guidati, G. and Wagner, S., "Experimental Demonstration of Wind Turbine Noise Reduction Through Optimized Airfoil Shape and Trailing-Edge Serrations", European Wind Energy Congress, Copenhagen, Denmark, July 2-6, 2001.

7. Roger, R. and Moreau, S., "Trailing Edge Noise Measurements and Prediction for Subsonic Loaded fan Blades", AIAA Paper 2002-2460.

8. Perennes, S. and Roger, M., "Aerodynamic Noise of a Two-Dimensional Wing with High-Lift Devices", AIAA Paper 98-2338.

9. Moreau, S. and Roger, R., "Effect of Airfoil Aerodynamic Loading on Trailing Edge Noise", AIAA Paper 2003-3177.

10. Hutcheson, F. and Brooks, T., "Measurement of Trailing Edge Noise Using Directional Array and Coherent Output Power Methods", AIAA Paper 2002-2472.

11. Brooks, T. F., Pope, D. S., and Marcolini, M. A., "Airfoil Self-Noise and Prediction", NASA Reference Publication 1218, July, 1989.
12. Oerlemans, S. and Sijtsma, P., "Determination of Absolute Levels from Phased Array Measurements Using Spatial Source Coherence", AIAA 20022464.

13. Brooks, T.F. and Humphreys, W.M., Jr., "Effect of Directional Array Size on the Measurement of Airframe Noise Components", AIAA Paper No. 99-1958, 1999.

14. Mendoza, J. M., Brooks, T. F. and Humphreys, W. M., "Aeroacoustic Measurements of a Leading Edge Slat Configuration", International Journal of Aeroacoustics, Vol. 1 (3), 2002.

15. Humphreys, W. M., Brooks, T. F., Hunter, W. W. and Meadows, K. R., "Design and Use of Microphone Directional Arrays for Aeroacoustics Measurements", AIAA Paper 98-0471, 1998.

16. Meadows, K. R., Brooks, T. F., Humphreys, W. M., Hunter, W. W., and Gerhold, C. H., "Aeroacoustic Measurements of a Wing-Flap Configuration”, AIAA Paper 97-1595, 1997.

17. Amiet, R. K.," Refraction of Sound by a Shear layer", Journal of Sound and Vibration, Vol. 58 (3), pp. 467-482, Sept. 1978.

18. Brooks, T. F. and Hodgson, T. H., "Trailing Edge Noise Prediction from Measured Surface Pressures", Journal of Sound and Vibration, Volume 78, Number 1, pp.69-117, 1981.

19. Brooks, T. F. and Marcolini, M. A., "Scaling of Airfoil Self Noise Using Measured Flow Parameters", AIAA Journal, Vol.23, 1985.

20. Brooks, T. F. and Marcolini, M. A., "Airfoil Tip Vortex Formation Noise", AIAA Journal, Vol.24, 1986.

21. Brooks, T. F., Marcolini, M. A. and Pope, D. S., "Airfoil Trailing edge Flow Measurements and Comparison with Theory Incorporating Open Wind Tunnel Corrections”, AIAA Paper 84-2266, 1984. 